\title{
Different effects of reward value and saliency during bumblebee visual search for multiple rewarding targets
}

\author{
Vivek Nityananda ${ }^{1,2}$ (D) Lars Chittka ${ }^{2}$
}

Received: 16 September 2020 / Revised: 11 January 2021 / Accepted: 13 January 2021 / Published online: 30 January 2021

(c) The Author(s) 2021

\begin{abstract}
Several animals, including bees, use visual search to distinguish targets of interest and ignore distractors. While bee flower choice is well studied, we know relatively little about how they choose between multiple rewarding flowers in complex floral environments. Two factors that could influence bee visual search for multiple flowers are the saliency (colour contrast against the background) and the reward value of flowers. We here investigated how these two different factors contribute to bee visual search. We trained bees to independently recognize two rewarding flower types that, in different experiments, differed in either saliency, reward value or both. We then measured their choices and attention to these flowers in the presence of distractors in a test without reinforcement. We found that bees preferred more salient or higher rewarding flowers and ignored distractors. When the high-reward flowers were less salient than the low-reward flowers, bees were nonetheless equally likely to choose high-reward flowers, for the reward and saliency values we used. Bees were also more likely to attend to these high-reward flowers, spending higher inspection times around them and exhibiting faster search times when choosing them. When flowers differed in reward, we also found an effect of the training order with low-reward targets being more likely to be chosen if they had been encountered during the more immediate training session prior to the test. Our results parallel recent findings from humans demonstrating that reward value can attract attention even when targets are less salient and irrelevant to the current task.
\end{abstract}

Keywords Attention $\cdot$ Bee $\cdot$ Flower constancy $\cdot$ Visual search

\section{Introduction}

Animal foraging behaviour is very well studied, but research in this area has not often considered more psychological aspects of foraging such as attention and visual search. Adapting human visual search experiments to investigate visual search in other animals, including bees, jays, owls and fish, has led to an increased understanding of their foraging behaviour and holds promise to become a productive field of research (Dukas and Kamil 2001; Bond and Kamil 2002; Spaethe et al. 2006; Morawetz and Spaethe

Vivek Nityananda

vivek.nityananda@newcastle.ac.uk

1 Biosciences Institute, Newcastle University, Henry Wellcome Building, Framlington Place, Newcastle Upon Tyne NE2 4HH, UK

2 School of Biological and Chemical Sciences, Queen Mary University of London, Mile End Road, Mile End, London E1 4NS, UK
2012; Nityananda and Pattrick 2013; Ben-Tov et al. 2015; Orlowski et al. 2015, 2018; Saban et al. 2017). Visual search experiments typically present individuals one target among distractors. Studies have also looked at how attention is deployed when more than one instance of a target type is present (Horowitz and Wolfe 2001) or how attention is divided across multiple tasks (Miller 1982). Fewer studies have looked at visual search for multiple object types or categories that are presented simultaneously (Duncan 1980; Huang et al. 2007; Kristjánsson et al. 2014; Berggren and Eimer 2020). Yet in real life we might well be searching for multiple items at a time, such as say, tomatoes and onions in the supermarket.

In bees, psychological studies of visual search have also focussed on how they choose single targets over others (Chittka and Spaethe 2007; Morawetz et al. 2015), and we know less about how they search in complex floral environments. Behavioural ecology research has focussed on flower constancy, the tendency of bees to specialize on a limited number of flower types out of those available (Heinrich 
1979; Wells and Wells 1983; Waser 1986; Hill et al. 1997). Bees can, however, learn multiple rewarding flower types (Nityananda and Pattrick 2013; Li et al. 2017) and readily approach these flowers, flexibly switching between at least two types (Nityananda and Pattrick 2013), although there can be temporal costs in switching between species, possibly reflecting working memory constraints (Raine and Chittka 2007). We still do not fully understand the role of attention and floral attributes in influencing bees' choices between multiple familiar flower types.

In humans, several factors are known to influence visual search (Wolfe and Horowitz 2004; Wolfe 2020), but two broad processes have typically been identified as fundamental. These are often classified as bottom-up and top-down visual search (Johnson and Proctor 2004). Bottom-up processes involve an involuntary, rapid capture of visual attention by salient stimuli. Top-down processes are more deliberate and guided by the goals of an immediate task. More recently, a third category of processes has been proposed involving the influence of search history (Anderson et al. 2011a; Awh et al. 2012; Anderson 2019; Theeuwes 2019). The most prominent examples of these processes have focussed on the role of reward value (Anderson et al. 2011a, b). Target stimuli that are relevant or monetarily rewarding in one task have been shown to capture visual attention even when they are irrelevant to a subsequent task and not salient (Anderson et al. 2011b; Bourgeois et al. 2017; Bucker and Theeuwes 2017). The capture of visual attention in these cases is also involuntary and rapid, as is typically seen in response to salient stimuli. Thus, visual search and attention can be influenced by three different processes dependent on physical saliency, current goals and search history.

The saliency of flowers as measured by their colour contrast against the background influences flower choice in bees (Lunau 1990; Lunau et al. 1996; Goulson 2000) and would also be expected to influence visual search and attention. Goal-driven visual search is more difficult to study in bees given the impossibility of providing verbal instructions to set goals for them. One way of specifying targets for the bees is to reward certain targets compared to others and reward value (sucrose concentration) does influence flower choice in bees (Benard et al. 2006; Avarguès-Weber and Giurfa 2014). However, this resembles reward-based visual search more than goal-directed search. Both reward and saliency could therefore influence visual search in bees. A bee might, however, simultaneously encounter flowers with differing saliency and reward and it is not yet known how these different factors could interact and influence visual search. In this study, we therefore ran a series of experiments to test how saliency and reward influence bee visual search for two simultaneously rewarding target types. We tested the idea that high-saliency targets would be preferred over equally rewarding low-saliency targets and that high-reward targets would be preferred over equally salient low-reward targets. We further tested the idea that when high-saliency targets were less rewarded than low-saliency ones, bees would preferentially attend to and choose the higher rewarding lowsaliency targets.

\section{Methods}

\section{Bees}

We obtained the bees from a commercial supplier (Syngenta Bioline, Weert, The Netherlands) and tagged them with Opalith number tags (Christian Graze KG, Weinstadt-Endersbach, Germany) to allow for individual identification. The bee colonies were transferred under red light to one chamber of a two-chambered wooden nest box $(28 \times 16 \times 11 \mathrm{~cm}$ length $\times$ width $\times$ height). The floor of the other chamber was covered with cat litter to give bees an area to discard refuse. The nest box was connected through a $24.5 \mathrm{~cm}$ long transparent Perspex tunnel to an arena consisting of a wooden box $(100 \times 60 \times 40 \mathrm{~cm}$ length $\times$ width $\times$ height $)$ covered with a UV-transparent Plexiglas lid. The bees could enter this arena to forage for sucrose solution. The floor of the arena was covered with green card and the illumination was provided from above using two twin lamps (TMS $24 \mathrm{~F}$ with HF-B 236 TLD (4.3 kHz) ballasts; Philips, The Netherlands) fitted with Activa daylight full spectrum fluorescent tubes (Sylvania, New Haven, UK). Pollen was provided directly into the colony on alternate evenings.

\section{Spectral measurements}

We measured the reflectance spectra of the artificial flowers using an Avantes AvaSpec 2048 spectrophotometer (Anglia Instruments Limited, Soham, UK) with a deuterium-halogen light source, relative to a $\mathrm{BaSO}_{4}$ white standard. To account for the difference between spectral sensitivity in humans and bees, we converted the spectra of the targets into a bee-specific hexagonal colour space (Chittka 1992) incorporating the spectral sensitivity of bumblebee photoreceptors (Skorupski et al. 2007), the spectral reflectance of the background and the spectral distribution of the lights used. The colour hexagon has three vertices corresponding to maximal excitation of each of the bee photoreceptors, which are tuned to green, blue and ultraviolet (UV) light (Chittka 1992). Three further vertices correspond to colour mixtures resulting from approximately equal excitation of two spectral receptors. The Euclidean distance between the centre of the hexagon and each of these vertices is 1 and colour distances greater than 0.1 are well distinguished by bees without special training procedures (Dyer and Chittka 2004a). Once plotted in this colour space (Fig. 1), the colour 
loci can be used to calculate the distances in colour space between pairs of colours, thus indicating the perceptual discriminability of the colours. All measures of colour differences between the artificial flowers used in our experiments are provided in Table S1.

\section{Pretraining}

We trained colour-naïve foraging bees to forage from square transparent Perspex chips (side: $25 \mathrm{~mm}$, thickness: $5 \mathrm{~mm}$ ) that served as artificial flowers (henceforth "flowers"). Each flower had a well in the centre into which rewarding (sucrose solution) or non-rewarding (water) liquids could be placed. After bees learned to approach these flowers, we placed them on glass vials $(4 \mathrm{~cm}$ tall, $1.5 \mathrm{~cm}$ in diameter) and trained the bees to feed from them when they were arranged in a $6 \times 4$ horizontal grid, with vials placed $15 \mathrm{~cm}$ apart. In this grid, 12 randomly chosen flowers had $12 \mu 1$ of $50 \%(\mathrm{v} / \mathrm{v})$ sucrose on them and the others remained empty. The positions of rewarding and non-rewarding flowers in all experiments were determined using the random number generator function RAND() in Microsoft $\operatorname{Excel}^{\circledR}$. Once the bees had foraged on this grid for three bouts, we commenced training.

\section{Training}

In each experiment, we trained bees from three different colonies on two visual discrimination tasks. The tasks involved bees having to discriminate target flowers of one colour from distractor flowers of another colour. The flowers consisted of coloured Perspex chips placed in a grid as described above. The 12 target and 12 distractor flowers were placed in positions on the grid that were randomly chosen for each bout of the training. Target flowers held $12 \mu \mathrm{l}$ of sucrose solution, while distractor flowers held $12 \mu \mathrm{l}$ of distilled water. Flowers were not refilled during a given training bout. Each bee was individually trained on one of these tasks until it reached a success criterion of $80 \%$ correct choices out of the last 20 choices made. Choices were recorded when the bee probed the flowers for reward and bees could revisit flowers in all experiments. Between training bouts, we cleaned the flowers with 99\% ethanol to remove scent markings (Pearce et al. 2017; MaBouDi et al. 2020), and subsequently with water to remove any traces of ethanol.

Once a bee successfully completed one training task, it was presented with another task consisting of target and distractor flowers with different colours from those in the first task. The order in which each of these tasks were presented was alternated between bees. The exact details of the colours
A

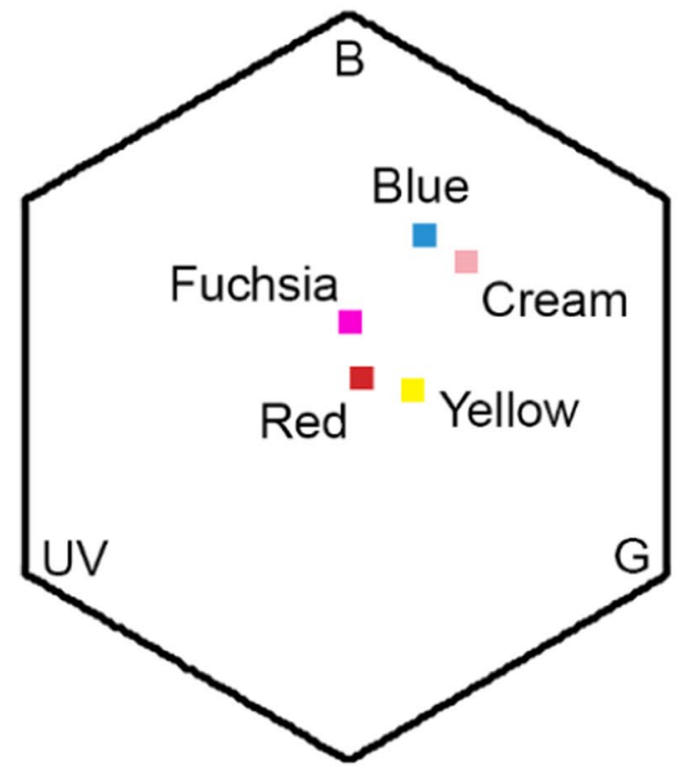

B

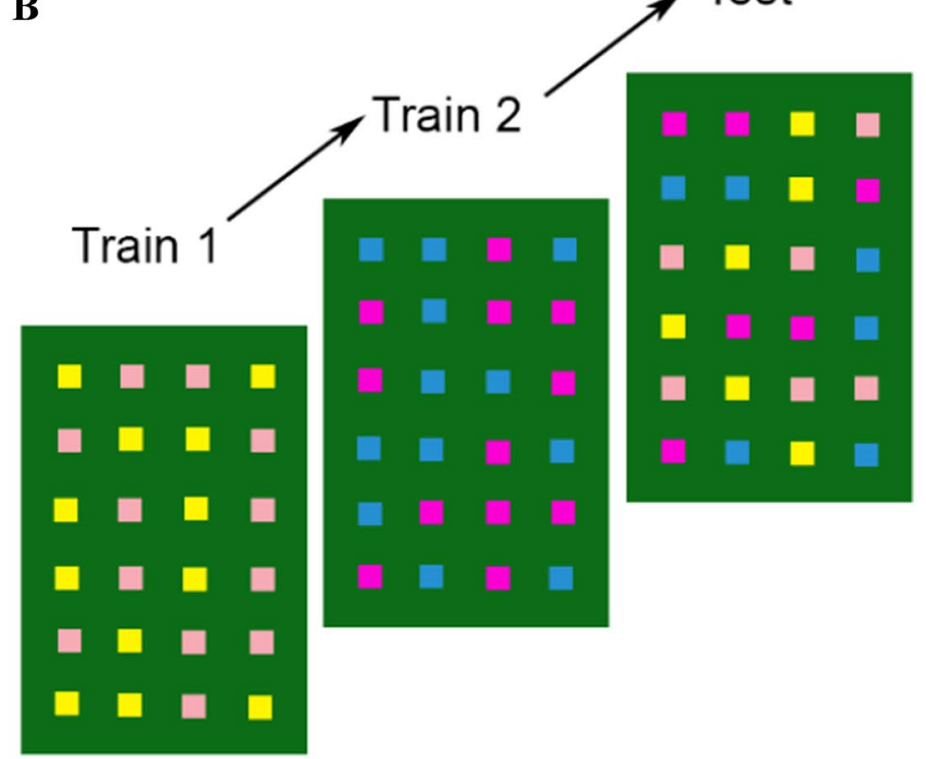

Fig. 1 a Colour loci of the artificial flowers used across all experiments in the colour hexagon. Three of the vertices correspond to maximum excitation of the bumblebee photoreceptors sensitive to ultraviolet (UV), blue (B) and green (G). The angular distance from the centre represents the hue as perceived by the bee. Distances between points indicate the hue discriminability. The dis- tance between the centre and any vertex is 1 and colours that differ by distances above 0.1 are easily distinguishable. b Example training and test protocol used in the experiments. Bees were trained on one rewarding and one non-rewarding colour in each training session (Train 1 and Train 2) and tested without reinforcement with all four colours in the test session (Test) 
and reward used are given below. Bees thus sequentially learnt two different rewarding colours.

\section{Experiment 1: How does saliency influence bee visual search?}

In this experiment, 20 bees were trained on 1 salient target and 1 less salient target in separate discrimination tasks. For ten of these bees, one of the two tasks had Blue as the rewarding colour and Cream as the non-rewarding colour. The other task had Fuchsia as the rewarding colour and Red as the non-rewarding colour. Both target colours provided a reward of 50\% sucrose solution (v/v). The experiment was replicated with another ten bees using a different set of colours. In this replication, the rewarding colours were Fuchsia and Red, while the distractors were Cream and Yellow, respectively. This replication ensured that Fuchsia, which was the less salient of the two target colours in the first combination was the more salient of the two target flower colours in the second combination of colours (Fig. 1). Time data are missing for one bee in this experiment because of the lack of video recordings.

\section{Experiment 2: How does reward value influence bee visual search?}

In this experiment, 15 bees were trained on 1 high-reward target and 1 low-reward target in separate discrimination tasks. One of these tasks had Blue as the rewarding colour and Fuchsia as the non-rewarding colour. The other had Cream as the rewarding colour and Yellow as the nonrewarding colour. These colours were chosen as the Blue and Cream colours were close in saliency, defined as colour contrast with the background (Table S1). In the two discriminations tasks, one of the target colours had a reward of 50\% sucrose solution (v/v) while the other had a reward of 30\% sucrose solution (v/v). With one exception, the association of high and low rewards with each of the target colours (Blue and Cream) was counterbalanced across all trials as was the order in which bees experienced high and low reward in their two training tasks. Time data are missing for five bees in this experiment because of the lack of video recordings.

\section{Experiment 3: How does bee visual search combine reward value and saliency?}

In this experiment, 16 bees were trained on 2 discrimination tasks. One of these had a high-reward target offering a reward of 50\% sucrose solution (v/v). This target was Yellow and had low colour contrast (i.e., saliency) against the background. The distractor in this task was Cream. In the other task, the target offered a lower reward of $30 \%$ sucrose solution (v/v). The target was Blue and had a high colour contrast against the background and the distractor was Fuchsia. The order in which bees encountered each of these tasks was counterbalanced.

\section{Test}

In all experiments, immediately after training was completed, we tested bees on their visual search when faced with multiple targets. All trained bees were tested, and the sample sizes were the same as mentioned above. We presented the bees with six flowers of each of the two rewarding colours they were trained on and six flowers of each of the two distractor colours. All flowers in the test were non-rewarding, containing $12 \mu \mathrm{l}$ of distilled water. This prevented reinforcement learning during the test. We noted the choices made by the bees, defined by probing of the flowers for reward, and the order they were made in. The foraging bout of each bee during the test was recorded using a Sony DCR-SR58E Handycam to enable later analysis of the times between the choices. We ran the tests until 5 minutes were over, or the bee returned to the colony after making at least 12 choices, whichever occurred sooner.

\section{Data analysis}

For all experiments, we split the choices made by the bees into the different transitions between colours and noted which were switches between flowers of different colours and which were constant transitions between two flowers of the same colour. We examined the number of constant transitions made before each switch to measure how often bees had runs of constant choices. We then calculated a sequence index for each bee by dividing the number of constant transitions by the total number of transitions (Heinrich 1979). This index is the probability of constant transitions compared to switches. An index close to 1 would indicate that the bees were flower constant while a value close to 0.5 would indicate that bees made an equal number of constant choices and switches. We used a Wilcoxon rank sum test $(\alpha=0.05)$ to compare the observed number of constant choices with the index values of 1 and 0.5 . We also examined how quickly bees made these different choices by comparing the median times taken to make constant choices and switches using Wilcoxon rank sum tests $(\alpha=0.05)$. Since the bees occasionally flew around the arena for extended periods of time without making a choice, prior to the second analysis, we ran an outlier analysis for the times within each category and excluded data points that were greater or less than 1.5 times the interquartile range.

To examine how different factors influenced the proportions of choices made by the bees, we ran generalized linear models with the proportion of choices as a dependent variable and the different factors as independent variables. 
For experiment 1, the independent variables were saliency (high or low) and a second variable representing the training order. This second variable was a binary variable representing whether the bee first encountered the high-saliency target or the low-saliency target during the training on visual discrimination tasks. For experiment 2 , the independent variables were reward value (high or low) and a second binary variable representing whether the bee first encountered the high-reward target or the lowreward target during training. For experiment 3, we also had the two independent variables as in experiment 2. In all the models, bee identity was modelled as a random variable and the proportion of choices was modelled as a binomial distribution with a logit link function. We ran models looking for main effects of the independent variables and interaction effects between the variables. In this and all other analyses, models were compared using the Akaike Information Criterion (AIC) and the model with the lowest criterion was chosen. The significance of each variable was compared against an $\alpha$ of 0.05 .

In experiment 3, we were also interested to see if higher reward could influence bee attention to a target with low saliency. We used the positions of the bee during visual search as a proxy for attention. Using the open-source program Tracker (V5.15, ${ }^{\circ} 2020$ Douglas Brown, physlets.org/ tracker), we perspective-corrected each video and tracked the position of the bee in each frame of the video during the test phase. We used this to analyse bee behaviour during the first 2 minutes of the videos. Frames in which it was not possible to spot the bee-either because it flew to the corner of the arena or due to reflections of the lighting-were labelled as missing data. Using the tracked positions of the bees we obtained a map of search behaviour for each bee. We specified zones on these maps corresponding to flower areas and non-flower areas. Flower areas were areas within $2 \mathrm{~cm}$ of the flowers. All other areas were non-flower areas. We measured inspection time defined as the sum of the number of video frames in which bees were present in each of these areas. The time in seconds would correspond to this value divided by the frame rate (25 frames per second). We compared the inspection time (defined as the summed number of frames) for the different types of targets and distractors. We used a generalized linear model to model this as a binomial variable with a logit link function. As in the analysis above we used reward value, saliency and search history as independent variables and bee identity as a random factor. We ran models looking for main effects of the independent variables and for interaction effects between the variables as well.

All statistical analyses were run in RStudio (version 1.2.5033).

\section{Results}

\section{Experiment 1: How does saliency influence bee visual search?}

The average time taken for the first and second training bouts on this experiment was $2080.7( \pm 1418)$ seconds and 971.9 $( \pm 366.4)$ seconds, respectively. Combining results from both flower sets, we found that the average proportion of salient target flowers chosen during tests was $0.58( \pm 0.13$ $\mathrm{SD})$ and the average proportion of equally rewarding nonsalient targets was 0.37 ( $\pm 0.11 \mathrm{SD})$. The average proportion of distractors chosen was $0.06( \pm 0.08 \mathrm{SD})$. If bees chose equally between the two targets without choosing any distractors, we would expect an equal proportion (0.5) of both salient and non-salient targets to be chosen. Saliency had a significant effect on the proportion of targets chosen; the proportion of high-saliency targets chosen was significantly greater than the proportion of non-salient targets chosen (GLMM, Effect size estimate: $-0.84, p=4.3 * 10^{-9}$, Fig. 2a) and the proportion of distractors chosen (GLMM, Effect size estimate $=-3.24, p<2 * 10^{-16}$, Fig. 2a). The low number of choices made to distractors demonstrates that the bees had memorised both types of previously rewarding targets in the training bouts and recalled them in the presence of distractors. The best model that described the data did not include the effect of training order, indicating that this was not an important determinant of the proportion of salient targets chosen.

The average sequence index of the bees was $0.51( \pm 0.17$ $\mathrm{SD})$. An index close to 0.5 indicates equal numbers of constant choices and switches, while an index close to 1 indicates complete flower constancy with no switches. This index was not significantly different from 0.5 (Wilcoxon rank sum test, $W=200, p=0.1$ ), showing that the bees were equally likely to make constant choices and switches (Fig. 3). The times taken for choices between like flowers and transitions between flower types were not significantly different (Wilcoxon rank sum test, $W=13,036, p=0.14$ ). The mean time taken for constant choices was $7.53( \pm 4.93$ S.D.) seconds compared to a mean of 9.03 ( \pm 7.05 S.D.) seconds for switches (Fig. 4a).

\section{Experiment 2: How does reward value influence bee visual search?}

The average time taken for the first and second training bouts on this experiment was $1033.8( \pm 439.8)$ seconds and 958.7 $( \pm 493.4)$ seconds, respectively.

The average proportion of high-reward targets chosen by bees was $0.69( \pm 0.18 \mathrm{SD})$, while the average proportion of low-reward targets chosen was $0.28( \pm 0.17$ 

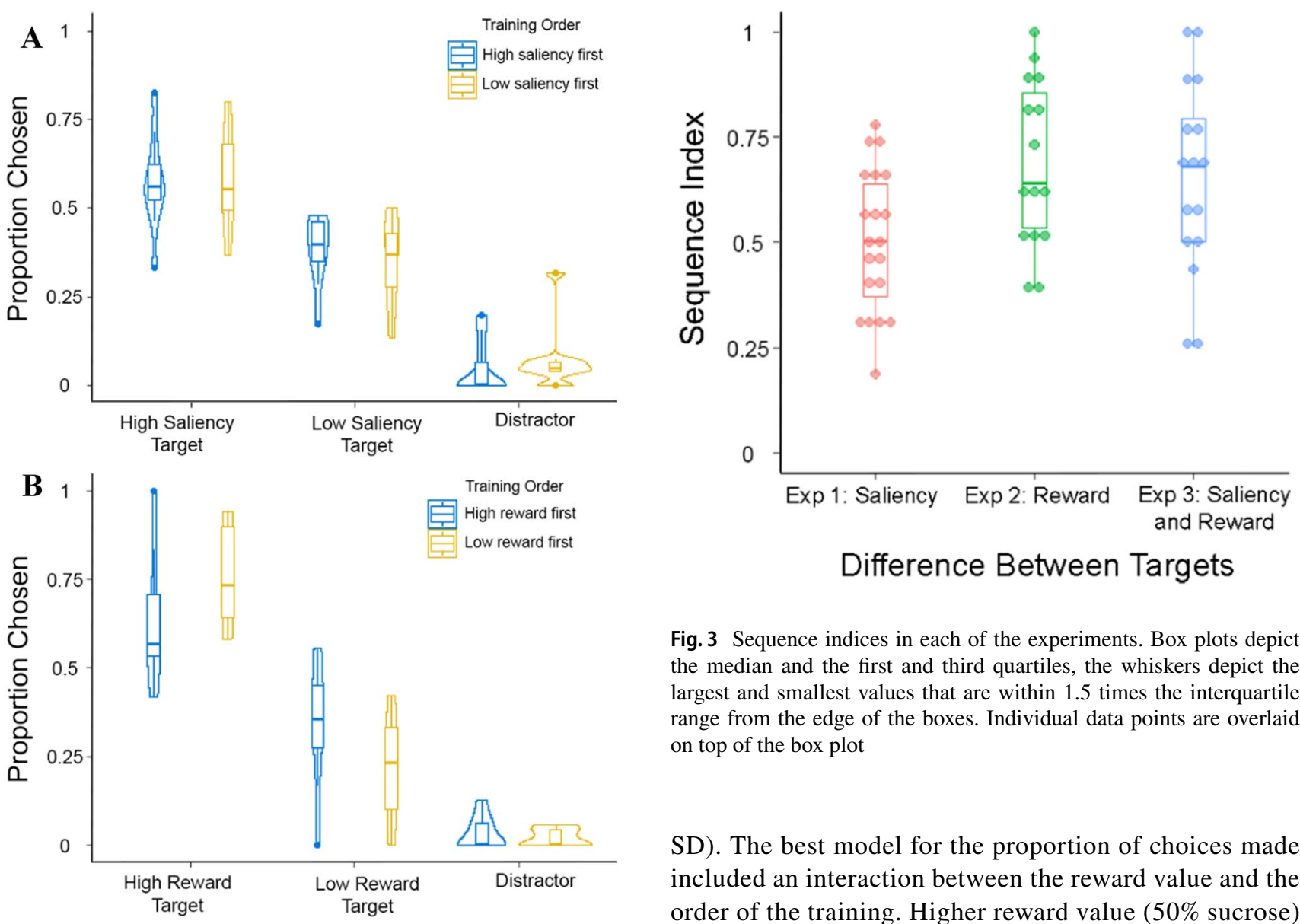

Fig. 3 Sequence indices in each of the experiments. Box plots depict the median and the first and third quartiles, the whiskers depict the largest and smallest values that are within 1.5 times the interquartile range from the edge of the boxes. Individual data points are overlaid on top of the box plot

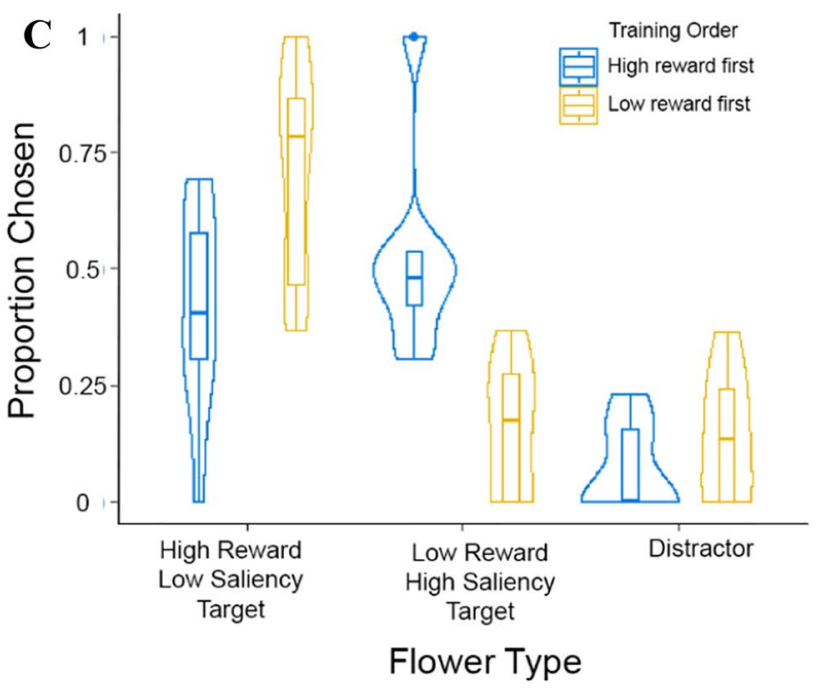

Fig. 2 Proportions of different flower types chosen in a experiment 1, b experiment 2 , and $\mathbf{c}$ experiment 3 . Box plots depict the median and the first and third quartiles, the whiskers depict the largest and smallest values that are within 1.5 times the interquartile range from the edge of the boxes. Violin plots overlaid on top of the box plot depict the mirrored density plots of the data. Note that the three proportions sum to 1 and so only two of them are free to vary
SD). The best model for the proportion of choices made included an interaction between the reward value and the order of the training. Higher reward value (50\% sucrose) led to a significantly greater proportion of choices compared to both low-reward (30\% sucrose) flowers (GLM, effect size estimate: $-1.12, p=2.15^{*} 10^{-5}$, Fig. 2b) and distractors (GLM, effect size estimate: -3.65 , $p=1.18 * 10^{-13}$, Fig. 2b). Thus, bees chose high-reward targets more often than low-reward targets. The average proportion of choices made to distractors was $0.02( \pm 0.04$ SD), demonstrating that the bees were capable of simultaneously choosing between two targets even in the presence of distractors.

Bees that were first trained on high-reward targets chose these targets significantly less than if they were first trained on low-reward targets (GLMM, effect size estimate: 0.72, $p=0.0088$ ). There was also a significant interaction effect between training order and reward value (GLMM, effect size estimate: $-1.38, p=0.0004)$. Bees were thus more likely to choose high-reward targets if they had been trained on them in the bout immediately preceding the test (i.e., trained on the low-reward targets first, yellow/right vs blue/left plots in Fig. 2b). The training times between the end of the first bout and the start of the test were, however, not significantly different when the first training bout had targets of high or low reward value (Wilcoxon rank sum test, $W=29, p=0.57$ ). The interaction effect between training order and reward value is thus not due to difference in training times. 

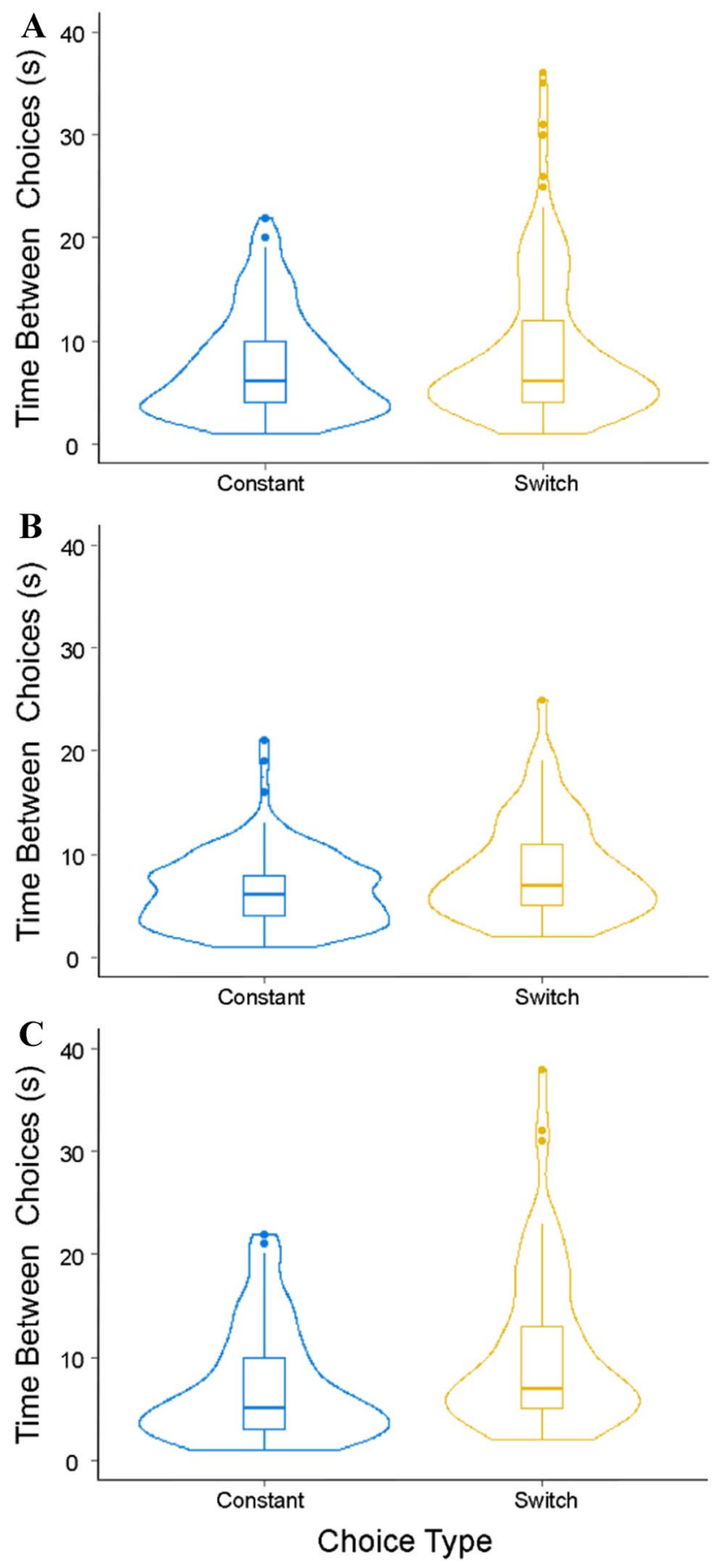

Fig. 4 Time taken to make constant choices and switches in a experiment $1, \mathbf{b}$ experiment 2 , and $\mathbf{c}$ experiment 3 . Box plots depict the median and the first and third quartiles, the whiskers depict the largest and smallest values that are within 1.5 times the interquartile range from the edge of the boxes. Violin plots overlaid on top of the box plot depict the mirrored density plots of the data

The average sequence index of the bees was $0.69( \pm 0.20$ SD) and this was significantly different from 0.5 (Wilcoxon rank sum test, $W=187.5, p=0.0008$, Fig. 3). This indicates that in this experiment, bees were more likely to have constant choices than switches. The time taken between choices was also significantly different between constant choices and switches chosen (Wilcoxon rank sum test, $W=2661.5$, $p=0.01$, Fig. 4b). The mean time taken for constant choices was $6.49( \pm 3.63 \mathrm{SD})$ seconds compared to a mean of 8.47 $( \pm 4.88 \mathrm{SD})$ seconds for switches.

\section{Experiment 3: How does bee visual search combine reward value and saliency?}

The average time taken for the first and second training bouts on this experiment was $1884.5( \pm 993)$ seconds and 1681.1 $( \pm 815.3)$ seconds respectively. The average proportion of high-reward, low-saliency targets chosen by bees was 0.56 $( \pm 0.27 \mathrm{SD})$, while the average proportion of low-reward, high-saliency targets chosen was $0.34( \pm 0.26 \mathrm{SD})$. There was no significant main effect of reward value on the proportion of high- and low-reward targets chosen (GLM, effect size estimate: $0.32, p=0.23$, Fig. 2 c) but a significantly higher proportion of high-reward targets were chosen compared to distractors (GLMM, effect size estimate: -2.42 , $p=2.55 * 10^{-8}$, Fig. $2 \mathrm{c}$ ). Thus, bees chose high-reward targets as often as low-reward targets, despite their lower saliency. The average proportion of choices made to distractors was low at $0.10( \pm 0.12 \mathrm{SD})$, demonstrating that the bees were capable of simultaneously choosing between two targets even in the presence of distractors.

The order in which bees were trained on the high-reward and low-reward targets had a significant main effect (GLM, effect size estimate: $1.1654, p=2.01 * 10^{-5}$ ). There was also a significant interaction effect between reward value and the order of the training (GLM, effect size estimate: -2.8688 , $p=3.39 * 10^{-12}$ ). Bees were thus more likely to choose highreward targets if they were the targets in the second training session (immediately prior to the test) rather than in the first training session.

The training times between the end of the first bout and the start of the test were, however, not significantly different when the first training bout had targets of high or low reward value (Wilcoxon rank sum test, $W=31, p=0.78$ ). The interaction effect between training order and reward value is thus not due to difference in training times.

The average sequence index of the bees was $0.65( \pm 0.25$ SD) and this was significantly different from 0.5 (Wilcoxon rank sum test, $W=192, p=0.0084$, Fig. 3). This indicates that in this experiment, bees were more likely to have constant choices than switches. The duration between choosing one flower and the next was also significantly different between constant choices and switches (Wilcoxon rank sum test, $W=3184, p=0.00053$, Fig. $4 c$ ). The mean time taken for constant choices was $7.14( \pm 5.36 \mathrm{SD})$ seconds compared to a mean of $10.51( \pm 7.78 \mathrm{SD})$ seconds for switches. 
The mean search time spent before choosing a highreward flower was $7.07( \pm 5.15 \mathrm{SD})$ seconds while the mean search time spent before choosing a low-reward flower was $9.51( \pm 7.33 \mathrm{SD})$ seconds, and these values were significantly different (GLM, Estimate $=-0.009, p=0.009$ ). Thus, the bees were quicker at choosing high-reward targets compared to low-reward targets. The model that best explained the proportion of time bees spent in different zones in the arena included flower type and the order in which bees were trained on high- or low-reward flowers as factors. Bees spent a significantly greater proportion of time around high-reward flowers than around low-reward flowers with greater saliency $\left(\right.$ GLMM, effect size estimate $=-0.63, p<2 * 10^{-16}$, Fig. 5a) and distractors (GLMM, effect size estimate $=-2.14, p<2 *$ $\left.10^{-16}\right)$. There was also a significant main effect of the order in which bees were trained on high- or low-reward flowers $\left(\mathrm{GLMM}\right.$, effect size estimate $\left.=0.84, p<2 * 10^{-16}\right)$ as well as an interaction effect between flower type and the order of training (GLMM, effect size estimate $=-2.28, p<2 *$ $10^{-16}$ ). Thus, when bees were trained on the high-reward flowers first and the low-reward flowers later, they were equally likely to spend time around high-reward, low-saliency flowers and low-reward high-saliency flowers. However, when trained on the low-reward flowers first and the high-reward flowers later, they spent a greater time around high-reward low-saliency flowers compared to low-reward high-saliency flowers.

B

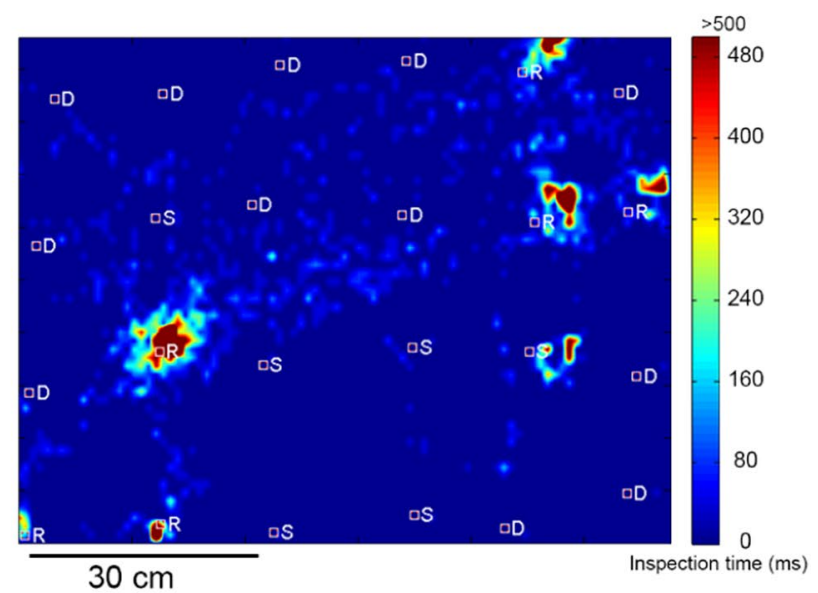

D

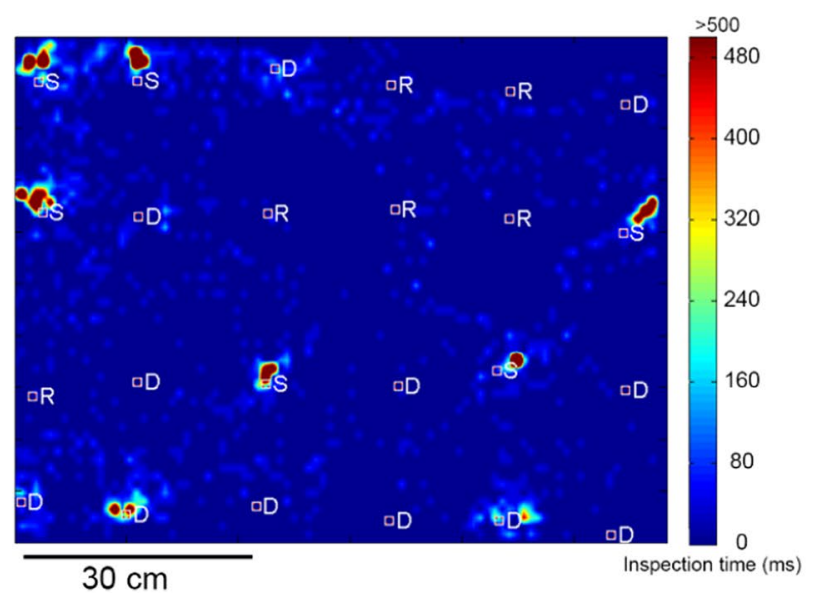

$R=$ High-reward, low-saliency targets; $S=$ Low-reward, high-saliency targets, $D=$ Distractors. B and C depict examples where bees spent more time around high-reward targets, D depicts an example where the bee spent more time inspecting low-reward targets 


\section{Discussion}

Bumblebees trained on multiple targets can choose the targets in the presence of distractors, without staying flower constant (Nityananda and Pattrick 2013). When targets are matched in both saliency and reward, bees are equally likely to choose either rewarding target, and switch between them often. Our results here demonstrate how bees can prioritize learnt rewarding targets when they differ in saliency, reward value or both. We found that differences in saliency and reward value did not hamper the visual search task, and bees in our experiment were still able to choose two target types and ignore distractors. Since the flowers were unrewarding during tests, the bees could in principle have changed their choices on not finding rewards and, for example, sampled more of the distractors or chosen both targets equally. We did not, however, find evidence for this in our results. In addition, in experiments 2 and 3, the distractors and the targets had a large colour distance (Fig. 1, Table S1) and this would have made it easier to avoid the distractors. However, in one of the two replications in experiment 1 , the targets and the distractors were relatively close in colour distance, but the bees were nonetheless able to avoid choosing the distractors.

Both saliency and reward influenced the proportion of targets chosen-with more salient and more rewarding targets chosen in higher proportions. This fits the classic model where the perceived value of the conditioned stimulus and the magnitude of the unconditioned stimulus both influence associative learning (Rescorla and Wagner 1972). The order in which bees encountered the targets during training also mattered when the targets differed in reward value and bees showed a recency effect (Ebbinghaus 1885). This was particularly evident when the targets differed in both saliency and reward value. While bees in this condition seemed to choose high-reward low-saliency targets at an equal proportion as low-reward high-saliency targets, a slightly different pattern was seen when training order was accounted for. Low-reward, high-saliency targets were more likely to be chosen if they were encountered in the most recent training bout rather than the earlier training bout. This effect was more pronounced for the high-reward, low-saliency targets. Our results also showed that high-reward targets led to greater flower constancy, shorter times for constant choices and more time spent attending to these targets.

Studies have long shown that bees can differentiate between targets of different colours that differ in reward value (Lubbock 1881; Turner 1910; von Frisch 1914; Benard et al. 2006; Avarguès-Weber and Giurfa 2014). Most studies, however, have typically used appetitive training paradigms where bees are trained to distinguish targets with a reward from distractors without a reward (Avarguès-Weber and Giurfa 2014). More recently, studies have focussed on aversive training paradigms where bees distinguish between targets that are rewarding and distractors that contain an aversive solution like quinine (Dyer and Chittka 2004b; Giurfa 2004; Avarguès-Weber and Giurfa 2014). These two approaches have different effects with aversive conditioning leading to more finegrained colour discrimination (Dyer and Chittka 2004b; Giurfa 2004). Studies that use two stimuli that are both rewarding but differ in reward value, as in this study, are fewer but they clearly demonstrate that bees can learn to differentiate colours even in this paradigm (Baude et al. 2011; Riveros and Gronenberg 2012; Avarguès-Weber et al. 2018). In one study using harnessed, rather than free-flying bees, the reward differential was provided by either providing the same concentration of sucrose solution to both the antenna and the proboscis (high reward condition) or to only the antenna (low reward condition). This differential was sufficient for bees to distinguish the colours associated with higher reward from those associated with lower rewards (Riveros and Gronenberg 2012). Our results from experiment 1 demonstrated that flowers that had a higher sucrose concentration were preferred by freely flying bees and biased their visual attention. The results from experiment 3 further showed that flowers previously associated with high reward were still chosen half an hour after the training, even when they had lower saliency than low-reward flowers.

The influence of saliency or colour contrast on bee visual search is less well studied than the influence of reward value (but see Spaethe et al. 2001). Some studies have looked at this in the context of the innate preferences of bees (Lunau 1990; Giurfa et al. 1995; Lunau et al. 1996). These preferences are typically biased towards the UVblue spectral range but do not seem to reflect the colour or green contrast difference from the background (Giurfa et al. 1995). Flower colours that have high spectral purity against backgrounds with low spectral purity do, however, attract the strongest innate behavioural responses from bumblebees (Lunau 1990). In addition, while bees can be trained to overcome their initial biases, their preferences can remain influenced by the effect of innate preferences (Gumbert 2000). In our experiment 3, we used a blue target as a low-reward target to see if the high reward value of the other target could overcome biases towards this target. We found this to occur if the bees were trained on the blue targets further in time from the test. Higher reward also biased visual attention away from the high-saliency blue targets as indicated by the time spent by the bees around different types of flowers. 
Our results also show that the search history of the bees is important to consider. Bees might often specialize on the first colour they find to be rewarding - regardless of saliency. This would prevent them from learning multiple targets as in our study. Other studies on honeybees have found persistent flower constancy when bees are not allowed to learn both targets independently (Wells and Wells 1983; Hill et al. 1997). In nature, multiple targets might possibly be learnt when floral communities are more diverse or have higher densities of flowers (Heinrich 1979; Chittka et al. 1997; Gegear and Thomson 2004; Baude et al. 2011). Our results further support the importance of reward value for constancy (Dukas and Real 1993; Greggers and Menzel 1993; Raine and Chittka 2007). Bees showed greater flower constancy when the targets differed in reward value. In these cases, they also took shorter times when making constant choices than when switching between colours. Bumblebees have been shown to fly shorter distances after visiting rewarding flowers compared to non-rewarding flowers (Dukas and Real 1993). We found that bees appeared more likely to switch between flowers that have equal reward value but stayed constant to highly rewarding flowers. Flower constancy is also affected by the density of conspecific bees (Baude et al. 2011), so including this along with reward value and floral diversity would make for a fuller picture of the ecology of flower constancy (Chittka et al. 1997).

Reward value also influenced the visual attention of the bees in addition to constancy and choice latencies. Bees spent a longer time inspecting high-reward flowers compared to low-reward flowers of greater saliency and were quicker to choose them. This resembles results from the human visual search literature, especially experiments demonstrating that the reward value associated with a stimulus can influence reaction times even if the stimulus is not task-relevant or salient (Anderson et al. 2011a, b). In our experiments we cannot assign task goals to the bees. However, the training order serves as a proxy for this. Half the bees in experiment 3 were initially trained on the high-reward target and then on the low-reward target. When faced with the test, the most recent training could arguably be considered the relevant task, making the previous high-reward targets irrelevant stimuli. Nonetheless bees still chose and attended to these targets-paralleling results in human experiments. We might potentially see different results when the reward values are lower, or the contrast of the high-reward target is reduced even further. When high-reward targets have very low detectability, low-reward targets with high physical saliency could have lower search times. In these cases, bees might then change their preference to low-reward targets rather than high-reward ones, especially if the rewards are not very different. The volumes of sucrose reward we used in our experiments were also higher than those seen in nature and it would therefore be important to see if our results hold even for more naturalistic volumes of reward. The use of different colour combinations different to the limited subset we use in our experiment would also be needed to confirm the generalizability of our results.

It has been argued that Pavlovian learning is important in reward-based attentional capture in humans, where the level of reward determines the effectiveness of attentional capture (Bucker and Theeuwes 2017; Mine and Saiki 2018). Since several well-studied animals, including bees, pigeons, starlings and rats, are well known for Pavlovian learning, it would be interesting to see if it could be involved in attention in other species as well. Our results suggest this might be true in bees and more focussed experiments testing if the mechanisms of attentional capture are shared across different animals would be an exciting area for future research.

Supplementary Information The online version contains supplementary material available at https://doi.org/10.1007/s10071-021-01479-3.

Acknowledgements $\mathrm{VN}$ is funded by a Biotechnology and Biological Sciences Research Council David Phillips Fellowship BB/S009760/1 and this work was partly carried out with support from a Marie Curie Incoming International Fellowship (PIIF-GA-2009-253593). LC is supported by EPSRC grant Brains on Board (EP/P006094/1). We thank Sara Khan, Muhammad Sayyidul Hasan and Piranavan Selvaratnam for help with running the experiments.

Funding VN is funded by a Biotechnology and Biological Sciences Research Council David Phillips Fellowship BB/S009760/1 and this work was partly carried out with support from a Marie Curie Incoming International Fellowship (PIIF-GA-2009-253593). LC is supported by EPSRC grant Brains on Board (EP/P006094/1).

Data availability Supporting data and scripts used to analyse and plot the data are available at https://doi.org/10.6084/m9.figshare.12907646.

\section{Compliance with ethical standards}

Conflicts of interest The authors have no conflicts of interest to declare that are relevant to the content of this article.

Ethical approval All applicable international, national, and/or institutional guidelines for the care and use of animals were followed. All procedures performed in studies involving animals were in accordance with the ethical standards of the institution or practice at which the studies were conducted.

Open Access This article is licensed under a Creative Commons Attribution 4.0 International License, which permits use, sharing, adaptation, distribution and reproduction in any medium or format, as long as you give appropriate credit to the original author(s) and the source, provide a link to the Creative Commons licence, and indicate if changes were made. The images or other third party material in this article are included in the article's Creative Commons licence, unless indicated otherwise in a credit line to the material. If material is not included in the article's Creative Commons licence and your intended use is not permitted by statutory regulation or exceeds the permitted use, you will need to obtain permission directly from the copyright holder. To view a copy of this licence, visit http://creativecommons.org/licenses/by/4.0/. 


\section{References}

Anderson BA (2019) Neurobiology of value-driven attention. Curr Opin Psychol 29:27-33. https://doi.org/10.1016/j.copsy c. 2018.11 .004

Anderson BA, Laurent PA, Yantis S (2011a) Value-driven attentional capture. Proc Natl Acad Sci. 108:10367-10371. https://doi. org/10.1073/pnas.1104047108

Anderson BA, Laurent PA, Yantis S (2011b) Learned value magnifies salience-based attentional capture. PLoS ONE 6:e27926. https:// doi.org/10.1371/journal.pone.0027926

Avarguès-Weber A, Giurfa M (2014) Cognitive components of color vision in honey bees: how conditioning variables modulate color learning and discrimination. J Comp Physiol A 200:449-461. https://doi.org/10.1007/s00359-014-0909-Z

Avarguès-Weber A, Lachlan R, Chittka L (2018) Bumblebee social learning can lead to suboptimal foraging choices. Anim Behav 135:209-214. https://doi.org/10.1016/j.anbehav.2017.11.022

Awh E, Belopolsky AV, Theeuwes J (2012) Top-down versus bottomup attentional control: a failed theoretical dichotomy. Trends Cogn Sci 16:437-443. https://doi.org/10.1016/j.tics.2012.06.010

Baude M, Danchin É, Mugabo M, Dajoz I (2011) Conspecifics as informers and competitors: an experimental study in foraging bumble-bees. Proc R Soc B Biol Sci 278:2806-2813. https://doi. org/10.1098/rspb.2010.2659

Benard J, Stach S, Giurfa M (2006) Categorization of visual stimuli in the honeybee Apis mellifera. Anim Cogn 9:257-270. https://doi. org/10.1007/s10071-006-0032-9

Ben-Tov M, Donchin O, Ben-Shahar O, Segev R (2015) Pop-out in visual search of moving targets in the archer fish. Nat Commun 6:6476. https://doi.org/10.1038/ncomms7476

Berggren N, Eimer M (2020) Attentional access to multiple target objects in visual search. J Cogn Neurosci 32:283-300

Bond AB, Kamil AC (2002) Visual predators select for crypticity and polymorphism in virtual prey. Nature 415:609-613. https://doi. org $/ 10.1038 / 415609 a$

Bourgeois A, Neveu R, Bayle DJ, Vuilleumier P (2017) How does reward compete with goal-directed and stimulus-driven shifts of attention? Cogn Emot 31:109-118. https://doi.org/10.1080/02699 931.2015.1085366

Bucker B, Theeuwes J (2017) Pavlovian reward learning underlies value driven attentional capture. Attention, Perception, Psychophys 79:415-428. https://doi.org/10.3758/s13414-016-1241-1

Chittka L (1992) The colour hexagon: a chromaticity diagram based on photoreceptor excitations as a generalized representation of colour opponency. J Comp Physiol A 170:533-543

Chittka L, Spaethe J (2007) Visual search and the importance of time in complex decision making by bees. Arthropod Plant Interact 1:37-44. https://doi.org/10.1007/s11829-007-9001-8

Chittka L, Gumbert A, Kunze J (1997) Foraging dynamics of bumble bees: correlates of movement within and between plant species. Behav Ecol 8:239. https://doi.org/10.1093/beheco/8.3.239

Dukas R, Kamil AC (2001) Limited attention: the constraint underlying search image. Behav Ecol 12:192-199. https://doi.org/10.1093/ beheco/12.2.192

Dukas R, Real LA (1993) Effects of recent experience on foraging decisions by bumble bees. Oecologia 94:244-246

Duncan J (1980) The locus of interference in the perception of simultaneous stimuli. Psychol Rev 87:272-300. https://doi. org/10.1037/0033-295X.87.3.272

Dyer AG, Chittka L (2004a) Biological significance of distinguishing between similar colours in spectrally variable illumination: bumblebees (Bombus terrestris) as a case study. J Comp Physiol A 190:105-114. https://doi.org/10.1007/s00359-003-0475-2
Dyer AG, Chittka L (2004b) Fine colour discrimination requires differential conditioning in bumblebees. Naturwissenschaften 91:224227. https://doi.org/10.1007/s00114-004-0508-x

Ebbinghaus H (1885) Über das Gedächtnis. Duncker and Humblot, Leipzig

Gegear RJ, Thomson JD (2004) Does the flower constancy of bumble bees reflect foraging economics? Ethology 110:793-805. https:// doi.org/10.1111/j.1439-0310.2004.01010.x

Giurfa M (2004) Conditioning procedure and color discrimination in the honeybee Apis mellifera. Naturwissenschaften 91:228-231. https://doi.org/10.1007/s00114-004-0530-z

Giurfa M, Nunez J, Chittka L, Menzel R (1995) Colour preferences of flower-naive honeybees. J Comp Physiol A 177:247-259

Goulson D (2000) Are insects flower constant because they use search images to find flowers? Oikos 88:547-552. https://doi. org/10.1034/j.1600-0706.2000.880311.x

Greggers U, Menzel R (1993) Memory dynamics and foraging strategies of honeybees. Behav Ecol Sociobiol 32:17-29. https://doi. org/10.1007/BF00172219

Gumbert A (2000) Color choices by bumble bees (Bombus terrestris): innate preferences and generalization after learning. Behav Ecol Sociobiol 48:36-43. https://doi.org/10.1007/s0026 50000213

Heinrich B (1979) " Majoring " and " minoring " by foraging bumblebees, bombus vagans : an experimental analysis. Ecology 60:245-255

Hill PSMM, Wells PH, Wells H (1997) Spontaneous flower constancy and learning in honey bees as a function of colour. Anim Behav 54:615-627. https://doi.org/10.1006/anbe.1996.0467

Horowitz TS, Wolfe JM (2001) Search for multiple targets: remember the targets, forget the search. Percept Psychophys 63:272-285

Huang L, Treisman A, Pashler H (2007) Characterizing the limits of human visual awareness. Science 80:823-825. https://doi. org/10.1126/science.1143515

Johnson A, Proctor RW (2004) Attention. Sage Publications, Thousand Oaks, California

Kristjánsson Á, Jóhannesson ÓI, Thornton IM (2014) Common attentional constraints in visual foraging. PLoS ONE 9:e100752. https ://doi.org/10.1371/journal.pone.0100752

Li L, Di MH, Egertová M et al (2017) A possible structural correlate of learning performance on a colour discrimination task in the brain of the bumblebee. Proc R Soc B Biol Sci 284:20171323. https:// doi.org/10.1098/rspb.2017.1323

Lubbock SJ (1881) Observations on ants, bees, and wasps. IX. Color of flowers as an attraction to bees: experiments and considerations thereon. Zool J Linn Soc 16:110-121

Lunau K (1990) Colour saturation triggers innate reactions to flower signals: flower dummy experiments with bumblebees. J Comp Physiol A 166:827-834

Lunau K, Wacht S, Chittka L (1996) Colour choices of naive bumble bees and their implications for colour perception. J Comp Physiol A 178:477-489. https://doi.org/10.1007/BF00190178

MaBouDi H, Solvi C, Chittka L (2020) Bumblebees learn a relational rule but switch to a win-stay/lose-switch heuristic after extensive training. Front Behav Neurosci 14:137. https://doi.org/10.3389/ fnbeh.2020.00137

Miller J (1982) Divided attention: evidence for coactivation with redundant signals. Cogn Psychol 14:247-279. https://doi. org/10.1016/0010-0285(82)90010-X

Mine C, Saiki J (2018) Pavlovian reward learning elicits attentional capture by reward-associated stimuli. Atten Percept Psychophys 80:1083-1095. https://doi.org/10.3758/s13414-018-1502-2

Morawetz L, Spaethe J (2012) Visual attention in a complex search task differs between honeybees and bumblebees. J Exp Biol 215:25152523. https://doi.org/10.1242/jeb.066399 
Morawetz L, Chittka L, Spaethe J (2015) Honeybees (Apis mellifera) exhibit flexible visual search strategies for vertical targets presented at various heights. F1000 Res https://doi.org/10.12688/ f1000research.4799.2

Nityananda V, Pattrick JG (2013) Bumblebee visual search for multiple learned target types. J Exp Biol 216:4154-4160. https://doi. org/10.1242/jeb.085456

Orlowski J, Beissel C, Rohn F et al (2015) Visual pop-out in barn owls: human-like behavior in the avian brain. J Vis 15:1-13. https://doi. org/10.1167/15.14.4

Orlowski J, Ben-Shahar O, Wagner H et al (2018) Visual search in barn owls: task difficulty and saccadic behavior. J Vis 18:1-13. https:// doi.org/10.1167/18.1.4

Pearce RF, Giuggioli L, Rands SA (2017) Bumblebees can discriminate between scent-marks deposited by conspecifics. Sci Rep 7:1-11. https://doi.org/10.1038/srep43872

Raine NE, Chittka L (2007) Flower constancy and memory dynamics in bumblebees (Hymenoptera: Apidae: Bombus). Entomol Gen 29:179-199. https://doi.org/10.1127/entom.gen/29/2007/179

Rescorla R, Wagner A (1972) A theory of Pavlovian conditioning: variations in the effectiveness of reinforcement and nonreinforcement. In: Black A, Prokasy W (eds) Classical conditioning II: current research and theory. Appleton Century Crofts, New York, pp 64-99

Riveros AJ, Gronenberg W (2012) Decision-making and associative color learning in harnessed bumblebees (Bombus impatiens). Anim Cogn 15:1183-1193. https://doi.org/10.1007/s1007 1-012-0542-6

Saban W, Sekely L, Klein RM, Gabay S (2017) Endogenous orienting in the archer fish. Proc Natl Acad Sci 114:7577-7581. https://doi. org/10.1073/pnas.1700574114

Skorupski P, Döring TF, Chittka L (2007) Photoreceptor spectral sensitivity in island and mainland populations of the bumblebee,
Bombus terrestris. J Comp Physiol Neuroethol Sensory Neural Behav Physiol 193:485-494. https://doi.org/10.1007/s0035 9-006-0206-6

Spaethe J, Tautz J, Chittka L (2001) Visual constraints in foraging bumblebees: flower size and color affect search time and flight behavior. Proc Natl Acad Sci 98:3898-3903. https://doi.org/10.1073/ pnas.071053098

Spaethe J, Tautz J, Chittka L (2006) Do honeybees detect colour targets using serial or parallel visual search? J Exp Biol 209:987-993. https://doi.org/10.1242/jeb.02124

Theeuwes J (2019) Goal-driven, stimulus-driven, and history-driven selection. Curr Opin Psychol 29:97-101. https://doi.org/10.1016/j. copsyc.2018.12.024

Turner CH (1910) Experiments on color-vision of the honey bee. Biol Bull 18:257-279. https://doi.org/10.1086/bblv18n5p213

von Frisch K (1914) Der Farbensinn und Formensinn der Biene. Zool Jahrb Abt Allg Zool Physiol Tiere 37:1-238

Waser NM (1986) Flower constancy: definition, cause, and measurement. Am Nat 127:593-603

Wells H, Wells PH (1983) Honey bee foraging ecology: optimal diet, minimal uncertainty or individual constancy? J Anim Ecol $52: 829-836$

Wolfe JM (2020) Visual search: how do we find what we are looking for? Annu Rev Vis Sci 6:1-24

Wolfe JM, Horowitz TS (2004) What attributes guide the deployment of visual attention and how do they do it? Nat Rev Neurosci 5:495-501. https://doi.org/10.1038/nrn1411

Publisher's Note Springer Nature remains neutral with regard to jurisdictional claims in published maps and institutional affiliations. 